\title{
Informal ties in organizations: a case study
}

\author{
Marco D'Errico · Silvana Stefani · Anna Torriero
}

Published online: 22 May 2013

C) Springer Science+Business Media Dordrecht 2013

\begin{abstract}
Network techniques are applied to a case study. The results show that using a joint approach can help in giving further insight into the analysis of informal ties in an organization. Special emphasis is given to centrality. The concept of mutual awareness, both on an individual and a global levels, is introduced and illustrated.
\end{abstract}

Keywords Organizational networks · Centrality measures · QAP

\section{Introduction}

Recent research has emphasized the degree to which information, that actors take action on, comes from other actors in their network. It has been made clear that the quality of an actor's network has a substantial impact on that actor's ability to solve problems, undertake projects, and implement plans (Anklam 2003). Essentially, people need strong networks to be successful over time. Furthermore, research shows that appropriate connectivity, in well-managed networks within organizations, can have a substantial impact on performance, learning, and innovation (Cross and Thomas 2008; Cross 2004; Anklam 2002, 2003; Cross and Cummings 2004). There are numerous active networks in an organization, both formal and informal. Discovering and analysing the hidden informal networks allows the identification and active engagement of those key individuals at all levels who can make the difference between

M. D’Errico ( $\varangle) \cdot$ S. Stefani

Department of Statistics and Quantitative Methods, University of Milano-Bicocca, Milan, Italy e-mail:m.derrico2@campus.unimib.it

S. Stefani

e-mail: silvana.stefani@unimib.it

A. Torriero

Department of Mathematics, Quantitative Finance and Econometrics, Catholic University, Milan, Italy e-mail: anna.torriero@unicatt.it 
success and failure. A positive approach to understanding informal networks can be the key in solving major organizational problems, identifying future organizational leaders and improving collaboration, both internally and externally. Whatever the central management imposes, informal networks develop in a way that can shape the work of the organization. Literature on informal networks is wide (see, for instance, Cross and Sproull 2004; Cross et al. 2001; Gibbons 2004; Krackhardt and Hanson 1993; Krackhardt and Stern 1988; Reagans et al. 2004). Social network analysis (SNA) can help the decision maker in modelling and understanding informal ties. This can be done, for instance, by checking the traffic of emails or phone calls to and from each node of the network or through specific surveys or ad hoc questionnaires. By individual questionnaires, the central management can generate network maps that will get to the root of many organizational problems (Brass 1984). In Cross et al. (2002) a research was conducted to determine how organizations can better support work occurring in informal networks of employees. The research was carried out through surveys on a large sample of companies. The results discussed the network topology and were largely based on degree centrality. In Brass (1984), a thorough analysis, based on results by questionnaires distributed to a sample of non-supervisory employees, was conducted. The study attempted to apply a structural perspective to the study of influence at the individual level. Activity and control of information were measured respectively by degree centrality and betweenness centrality. In Anklam (2003) and Cross (2004), using the concept of hubs and authorities (Farahat et al. 2006), the flow of information was modelled and bottlenecks or the sources of inefficiency in communication were detected.

Information and collaboration networks can be analyzed separately, but it is believed that the complexity of relationships among people can be best captured through multiple networks.

Following Robins and Pattison (2006), a multiple social network is a set of $n$ social actors and $k$ relations specifying how the actors are mutually linked. Each of the $k$ relations defines a univariate network on the actors (Wassermann and Faust 1994). The analysis of univariate networks is rich, both from an empirical and a theoretical point of view. For theoretical contributions to the analysis of eigencentrality in univariate networks see Grassi et al. (2007). There are several empirical studies (see, for instance, Naimzada et al. 2009, Newman 2010). On the other hand, multiple networks studies are quite rare, both theoretically and empirically.

For definitions and properties see Robins and Pattison (2006). Some applications can be found in De Toni and Nonino (2010).

Some studies deal with a multiple network as a whole or two-mode three-way data, using multidimensional scaling (Okada and Imaizumi 2002; Okada et al. 2005). Statistical techniques are proposed for analyzing jointly or comparing couples of univariate networks (Krackhardt 1987, 1988). In general, the two-mode three-way approach is considered preferable when dealing with multiple networks. However, since the focus is on centrality, that is originally defined in the context of a single network, it is not at all evident how to apply this concept to multiple networks jointly. To this aim, other methods are typically applied to each univariate network and then compared network by network. This approach is commonly used in identifying the central actors in organizational networks throughout computation of centrality measures for each node in a single network and then comparing the results (Krackhardt 1992a). In this paper, following the approach by Krackhardt and Hanson (1993), Krackhardt (1992a), a case study is analyzed and the informal networks in advice, communication and trust are mapped. Focusing in particular on the intersection and union networks, the central actors are identified. Special attention is given to mutual (or bilateral) awareness, i.e. the perception of giving help and advice as perceived by the recipient. In other words, if $A$ claims to give advice to $B$ but $B$ does not perceive to receive advice from $A$, the level 
of communication and understanding between $A$ and $B$ is not good. ${ }^{1}$ Mutual awareness is measured both at the individual level and the global level, i.e. to the organization as a whole.

In Sects. 2 and 3 the preliminaries and methodology are described. In Sect. 3 the methodology related to univariate networks is contrasted with the multiple networks method. In Sect. 4 the methodology is described and applied to the case study of a small consulting company reorganization. Section 5 concludes.

\section{Preliminaries}

We quickly recall some standard definitions and results about graph theory. We will assume familiarity with basic graph-theoretic concepts (see Harary 1969). A graph $G=(V, E)$ consists of a set $V$ of $n$ vertices and a set $E$ of edges. Let's denote with $|V|$ and $|E|$ the cardinality of the sets $V$ and $E$, respectively. Moreover, a weight $w_{i j}$ is possibly associated to each edge $(i, j)$, in this case there will be a weighted (or valued) graph.

The degree $d_{i}$ of a vertex $i(i=1, \ldots, n)$ is the number of edges incident to it. In a directed graph the in-degree of a vertex $i$ is the number of arcs directed from other vertices to $i$ and the out-degree of a vertex $i$ is the number of arcs directed from $i$ to other vertices.

A graph is connected if for each pair of nodes $i$ and $j(i, j=1,2, \ldots, n)$, there is a path from $i$ to $j$. A digraph is strongly connected if for each pair of vertices there is a directed path.

A non-negative $n$-square matrix $A=\left[a_{i j}\right],(i, j=1,2, \ldots, n)$ representing the adjacency relationships between vertices of $G$ is associated to the graph (the adjacency matrix); the off-diagonal elements $a_{i j}$ of $A$ are equal to 1 if vertices $i$ and $j$ are adjacent, 0 otherwise. Let $\left\{\lambda_{1}, \lambda_{2}, \ldots, \lambda_{n}\right\}$ be the set of the eigenvalues of $A, \rho=\max _{i}\left|\lambda_{i}\right|$ its spectral radius; if $G=(V, E)$ is undirected, it is known that $A$ is symmetric and its eigenvalues are real. When $G=(V, E)$ is connected, $A$ is irreducible, i.e. for every pair of indices $i$ and $j$, there exists a natural number $m$ such that $\left(A^{m}\right)_{i j} \neq 0$. If $G=(V, E)$ is a digraph, its adjacency matrix $A$ is in general asymmetric; moreover, $A$ is irreducible if and only if $G$ is strongly connected. If $A$ is a non-negative and irreducible matrix, it is well known that a positive eigenvector, called the Perron or principal eigenvector, corresponds to the principal eigenvalue of $A$.

In the case of a weighted graph, the adjacency matrix, denoted by $W$ (the weighted adjacency matrix) has zero diagonal elements, and non-negative off-diagonal entries.

\section{The methodology}

This section illustrates the ways in which informal organizational networks can be analyzed in order to identify the key actors in an organization. We focus in particular on centrality (Cross et al. 2002). The analysis starts with the case of univariate networks and concludes with multiple networks.

\subsection{Univariate networks}

The main centrality measures applied to a univariate network are reviewed here. The concept of degree centrality is recalled first. Degree centrality has an immediate interpretation in an

\footnotetext{
1 Our concept of awareness differs from Chen et al. (2007) or Cross and Cummings (2004). Awareness is defined in Chen et al. (2007) as a competitor's operating capacity compared with that of a focal firm. Awareness of another's expertise in Cross and Cummings (2004) is the extent to which one person knows what another person knows.
} 
organizational network, i.e. the degree of a person is the number of individuals to which he/she communicates directly. For directed networks, to which asymmetric adjacency matrices are associated, the degree centrality is replaced by in- and out-degree centrality that can be interpreted in terms of popularity and expansiveness, respectively.

A centrality measure enhancing the importance of a node as a function of centrality of the nodes to which it is connected, is the eigenvector centrality (see Bonacich 1987, 2007): the centrality of an actor is proportional to the sum of the centralities of the neighbours. Formally, the eigencentrality $x_{i}$ of the vertex $v_{i}$ is defined as $x_{i}=k \sum_{j=1}^{n} a_{i j} x_{j}$; by setting $k=\frac{1}{\rho}$, the value $x_{i}$ is represented by the $i$-th component of the Perron eigenvector $\mathbf{x}$ (see Grassi et al. 2007 for a review on eigencentrality and further results).

In formal or informal organizational networks, the relations between actors are in general directed, so that the corresponding graph is a digraph and the adjacency matrix is asymmetric. Accordingly, the eigenvalue centrality has to be modified to take into account this asymmetry. Two attributes are given to each node: authority and hubness (Kleinberg 1999). Authority measures prestige: actors who many other actors point to are called authorities. If a node has a high number of nodes pointing to it, it has a high authority value and that quantifies its role as a source of information. On the contrary, a hub is an actor referring to many authorities and its score measures acquaintance. Essentially, a good hub points to many good authorities and a good authority is pointed to by many good hubs.

More formally, let $G=(V, E)$ be the directed graph on $n$ nodes modelling the network and $A(G)$ the asymmetric adjacency matrix of $G$ of order $n$. Two scores (centralities) are associated to each node: $x_{i}$, the authority score for node $i$ and $y_{i}$ the hub score for node $i$. These values are reciprocally defined in a recursive scheme that is the basis of HITS (Hyperlink-Induced Topic Search) algorithm (see Kleinberg 1999). HITS leads to authorities and hubs as the (normalized) principal eigenvectors $\mathbf{x}^{*}$ and $\mathbf{y}^{*}$ of the symmetric semi-positive definite matrices $A^{T} A$ and $A A^{T}$, where vectors $\mathbf{x}=$ $\left[x_{1}, \ldots, x_{n}\right]^{T}$ and $\mathbf{y}=\left[y_{1}, \ldots, y_{n}\right]^{T}$ give respectively the authority and the hub scores on all nodes.

The betweenness of a vertex $v$ (see Freeman 1977), is defined, for both undirected and directed graphs, as the percentage of shortest paths between pairs of vertices passing through $v$; for every $u, w \neq v, b(v)=\sum_{u<w} \frac{g_{u w}(v)}{g_{u w}}$, where $g_{u w}$ is the number of geodesics from $u$ to $w$, and $g_{u w}(v)$ is the number of geodesics between $u$ and $w$ passing through $v$, (see Grassi et al. 2010a for an application of betweenness to an organizational network and Grassi et al. 2009 for some theoretical results). It is convenient to normalize the betweenness dividing $b(v)$ by its maximum possible value $\left(\begin{array}{c}n-1 \\ 2\end{array}\right)$, so that the normalized betweenness lies in the interval $[0,1]$.

Finally, following the approach introduced by Okada (2010) where hub and authority scores are represented in the bidimensional plane, each person in the organization is represented by a dot in the $x-y$ plane: the intensity (normalized eigencentrality) of each person in contacting other people to be helped in problem resolution (hub) is read on the $x$ axis, while the intensity (normalized eigencentrality) of the person being contacted by other people to solve problems (authority) is read on the $y$ axis.

The bisecting line $x=y$ is the boundary between hubs and authorities. Dots above this line indicate people who act more as authorities rather than hubs, while the opposite is the case below that line. Dots close to the line are at the same time givers and takers, and show a good level of communication, mutual help and contact with other people. 


\subsection{Multiple networks}

With reference to the case study, ten types of relations $(k=10)$, identifying ten single networks (Information network, Advice network, ...), are dealt with. In the presence of $k$ different types of relations between $n$ actors, there are $2^{k}$ different possible ties joining actors $i$ and $j(i, j=1, \ldots, n)$. Let $a_{h}(i, j)=1$ if there is a relationship of type $h$ from actor $i$ to actor $j$ and $a_{h}(i, j)=0$, otherwise. The analysis of a multiple network is currently carried out in different ways. One method analyzes the many networks jointly, basing on multidimensional scaling, This allows to analyze the difference among different matrices simultaneously, using models based on distance (Okada and Imaizumi 2002; Okada et al. 2005). Following another approach, we computed the intersection and union networks (Krackhardt 1992a; Robins and Pattison 2006; De Toni and Nonino 2010). In the intersection network, actors $i$ and $j$ are linked if and only if $\forall h, a_{h}(i, j)=1$. The adjacency matrix $A_{\cap}$ of the intersection network can be easily obtained through the Hadamard product $A_{\cap}=$ $A_{1} \circ A_{2} \circ \cdots \circ A_{k}$, that is the entry-wise product, between the $k$ adjacency matrices of the corresponding univariate networks. The intersection network allows the discovery of strong ties within the organization (Krackhardt 1992a), as far as help and advice are concerned. Analogously, in the union network the presence of a link between actors $i$ and $j$ means that they are connected at least in one of the $k$ networks. The adjacency matrix of the union network $A_{\cup}$ is obtained by summing up $A_{1}, A_{2}, \ldots, A_{k}$ and binarizing the resulting matrix. The union network allows to check the presence of any possible link between actors from exchanging simple information to substantial advices.

The "bisecting line" procedure can also be extended to the analysis of a couple of networks for providing a measure of the degree of awareness of a person into the organization (mutual awareness). In fact, as is shown later, by coupling networks corresponding to a given question, asked both directly and indirectly, and plotting hub and authority scores, those individuals who are perfectly integrated within the organization can be identified. Bad levels of communication and understanding among people are revealed depending on the position above or below the bisecting line. Mutual awareness at a global level can be analyzed through the Quadratic Assignment Procedure (QAP) (Krackhardt 1987, 1988, 1992b). QAP is used to compare couples of univariate networks. In our case, information and collaboration networks are compared considering the two sides of respondents, i.e. who gives advices and help and who receives it. The comparison will allow to understand the level of awareness in collaboration for the organization as a whole.

\section{The case study}

A family-owned consulting company which has to deal with leadership transition is presented. The owner wants to retire and leave control to heirs, part of whom are involved directly in the management.

The small sized company ${ }^{2}$ has always been characterized by flexibility and the ability to quickly adjust to change. People working in the front office area have been left free to establish contacts with clients and assist them from the beginning to the end. On the one hand, people were motivated in pursuing the interests of the company, on the other hand this granted too much freedom and gave the opportunity to some brilliant and ambitious consultants (Frank)

2 The company is composed of: Ted (the owner); Alex and Anna (his daughters), Charly (Alex's husband), Frank and Coch (consultants close to the family), Chin, Ary, Dominic, Four, Dellor, Taggy (external consultants), Jac (a new employee), Joan and Laura (secretaries), Sara (psychologist). 
to manage too much information within the organization and even to attempt a sort of take over. Thus, a restructuring process is needed, to allow a smooth transition and to give the necessary power back to family members.

After extensive interviews and talks, a new formal organization of the company was proposed and subsequently accepted by Ted. However, after restructuring, the comitant still feels that a more thorough analysis on the informal relationships within the organization should be performed. The flow of information is probably not efficient and the informal ties have to be discovered. The informal should be adapted to formal, or viceversa, through specific actions. If the analysis shows that the leading and crucial roles in the organization chart are assigned to people who are collaborative, give advice and understand the needs of others, then the whole organization is efficient. If, on the other hand, it is discovered that some sectors do not interact effectively, then intervention is required. In synthesis, the restructuring process can be divided into three phases:

1st Phase: formal restructuring,

2nd Phase: understanding informal ties,

3rd Phase: decision.

The first phase is described in detail in Grassi et al. (2010a). This paper describes the instruments, methodology and results related to the second phase. Preliminary results can be found in Stefani and Torriero (2013).

\subsection{The questionnaire}

Following Anklam $(2002,2003)$ and Cross (2004), a short questionnaire including ten questions, $Q_{i}, i=1, \ldots, 10$, was distributed to the people in the company, Ted included.

The six possible answers range from 1 to 6 , according to various levels of connections, from the weakest to the strongest. More precisely, if the people interact rarely, then we set $1=$ very seldom, $2=$ seldom, $3=$ fairly seldom, while for frequent contacts: $4=$ fairly frequently, $5=$ frequently, $6=$ very frequently. Null responses receive 0 score.

Essentially, we asked each person to rate his/her interactions with other members of the group, according to different levels of acquaintance. We checked for relationships that revealed collaboration or mistrust (e.g. information, problem solving, decision making, creation of value, mistrust). With the invaluable help of Sara, the company psychologist, who was herself interviewed, the "dual" of each question was added. In other words, we checked not only for the various levels of acquaintance but also how the contact by one person was perceived by the recipient, i.e. if for instance a request for help before making a decision was perceived as such by the other. In other words, mutual awareness was checked for.

- How often do you receive information from $\left(Q_{1}\right)$, or give information to $\left(Q_{6}\right)$ this person to accomplish work?

These questions check for frequency of direct contacts and whether or not people are aware of each other's knowledge and skills.

- How often do you contact $\left(Q_{2}\right)$ or are contacted by $\left(Q_{7}\right)$, this person to get (or give) help and advice to solve a problem at work?

These questions check for the willingness of people to help others and be helped.

- How often do you contact $\left(Q_{3}\right)$ or are contacted by $\left(Q_{8}\right)$ this person before making a decision?

These questions check for the expertise network and for strong ties at work. This reveals the strategic information sharing potential. 
- How much time was saved last month by contacting $\left(Q_{4}\right)$ or being contacted by $\left(Q_{9}\right)$, this person and receiving or giving help and advice?

These questions check for creation of value and for the key contributors in the organization along with a baseline time value that can be used for future metrics and evaluation.

- How often do you get the feeling that this person is not sharing useful information with you $\left(Q_{5}\right)$ ? How often, for various reasons, do you not share useful information with this person $\left(Q_{10}\right)$ ?

These questions check for possible delays in the information flow. The level of trust is investigated.

Due to different purposes, for each question, two matrices are obtained. One is the weighted adjacency matrix, with the scores from the questionnaire. The other matrix is a binary adjacency matrix, obtained by grouping scores into two categories according to the intensity of interactions: rare or null: $0=0-3$; frequent: $1=4-6$. Note that the current grouping has been used after testing more options. Results do not differ substantially.

Hence, the binary adjacency matrix corresponding to the $i-t$ question is denoted by $Q_{i}^{b},(i=1, \ldots, 10)$ while the weighted adjacency matrices by $Q_{i}^{w},(i=1, \ldots, 10)$.

\subsection{Results and comments}

\subsubsection{Univariate and multiple centrality}

In order to discover the most central actors, we properly refer to the binary adjacency matrices. In fact, most centrality measures, considered in our analysis, are commonly defined only for binary data (Bonacich 1987, 2007; Wassermann and Faust 1994). Clearly, this could entail a loss of original information contained in the questionnaires. However, as far as the importance of an actor is concerned, the score of the interaction is not particularly relevant so that the binarization seemed reasonable.

Degree (in/out), eigen- (hub/authority) and betweenness centralities are obtained for each question, i.e. for each univariate and then for the multiple networks, corresponding to the Hadamard product and union of $Q_{1}^{b}, Q_{2}^{b}, Q_{3}^{b}, Q_{4}^{b}$. A detailed analysis of question $Q_{5}$ is found in Stefani and Torriero (2013). High in-degree centrality identifies a "prominent" or a "popular" actor, i.e. a central connector. Information brokers are the betweenness central actors. They play a brokering role that can hold together entire groups. Peripheral specialists have sparse direct connections and may suffer from isolation (Cross and Thomas 2008; De Toni and Nonino 2010).

Actors with a high eigenvector centrality (authorities) can be candidates for leadership in the company, so they are called drivers (Naddaf and Mutyala 2010).

The intersection network shows the systematic request of help or advice to (or from) specific individuals and this reveals the strength of ties. On the other hand, low centrality in the union network shows a lack of permanent relationships.

The intersection network From the Hadamard product of $Q_{i}^{b}(i=1, \ldots, 4)$, in- and outdegree centrality were first checked for. In Figs. 1 and 2, the in- and out-degree rankings for the networks associated to questions $Q_{i}(i=1, \ldots, 4)$ and their intersection are reported respectively. Individuals are listed from the highest to the lowest (in- or out-) degree score.

Charly is by far the authority central connector. His position is strategic for the organization, both for simple information and for substantial help. Frank is an authority as well but not 


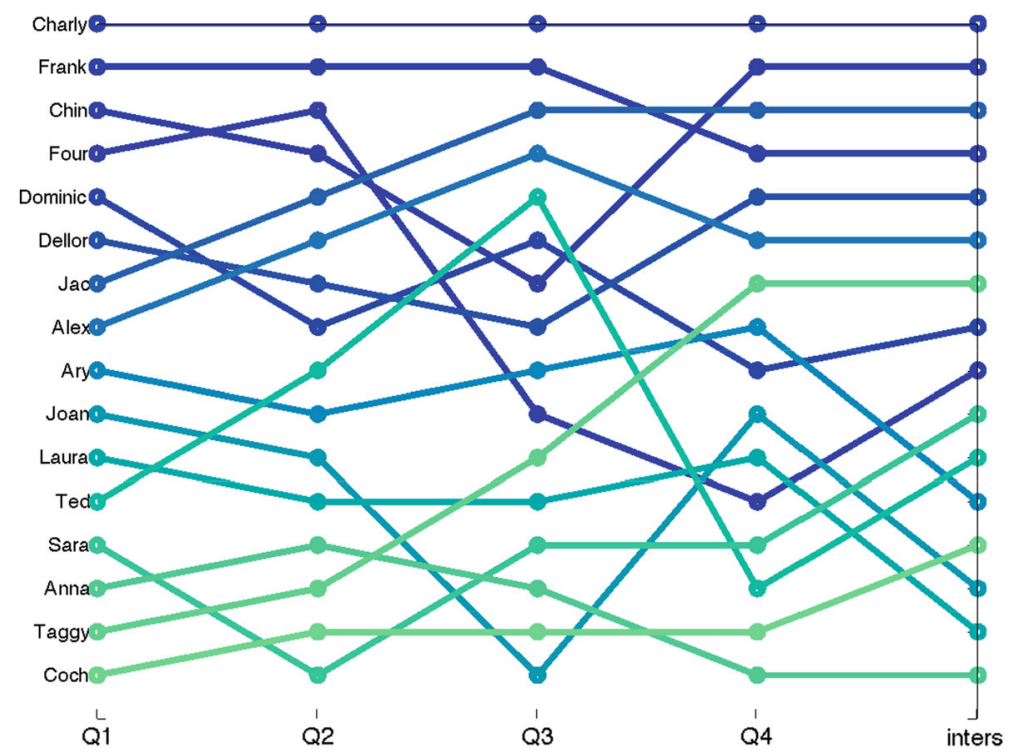

Fig. 1 Intersection: in-degree rankings

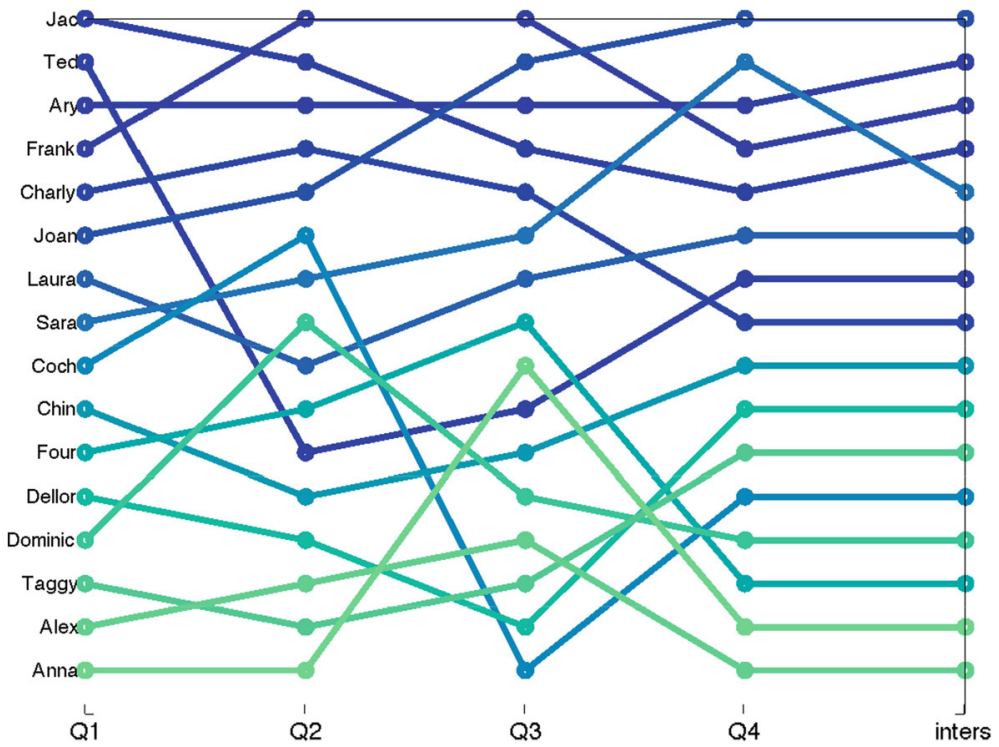

Fig. 2 Intersection: out-degree rankings

for important advice. Chin helps people in saving time and provides valuable information. Dellor and Jac become central as soon as decision making and key advice are involved. Alex keeps an intermediate position. Note that Charly is a top manager, Jac is a head of the back office, while Dellor and Chin are external consultants, so it seems that the key helpers are well distributed within the organization. As far as the President (Ted) is concerned, he is contacted mainly before making important decisions (question $Q_{3}$ ). From the side of "askers", 


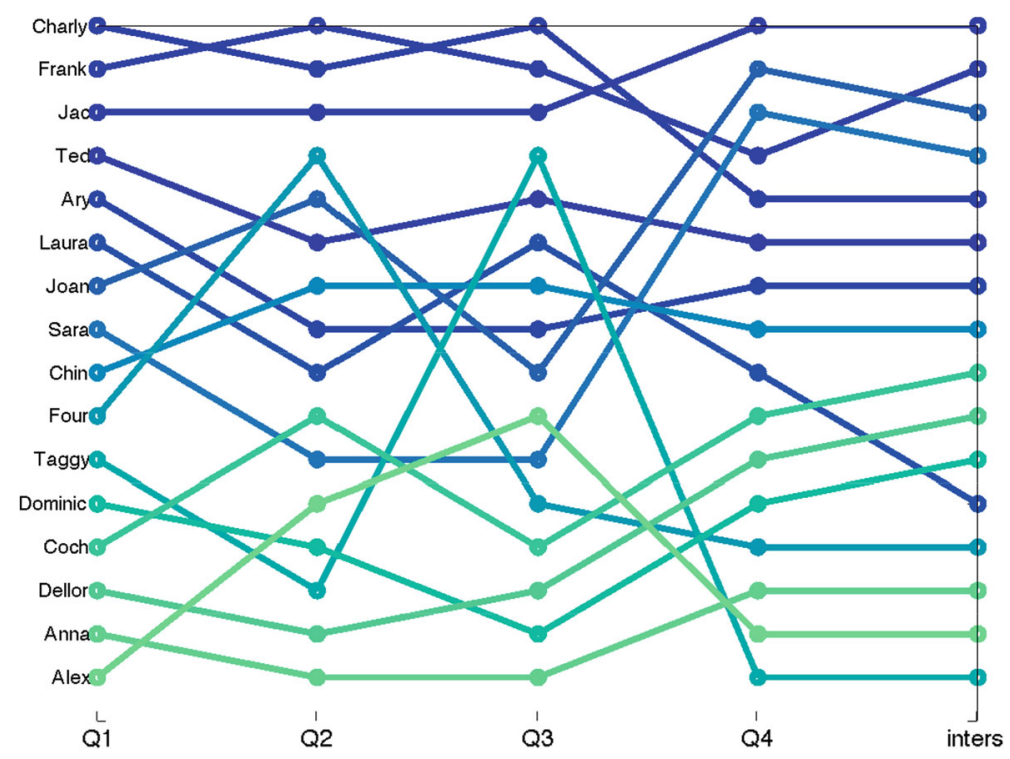

Fig. 3 Intersection: betweenness rankings

Joan, one of the secretaries, gets the highest score: given her doubtful personality, she needs confirmation before making any decision. Frank, Jac and Ary often ask for advice and help. Charly is a moderate asker. On the other hand, it seems that Alex does not need any help, especially for important decisions. As far as the betweenness ranking is concerned (Fig. 3), Jac, Charly and Frank play central roles.

Results on eigencentrality for hubs (not reported in figures) confirm what has been found for out-degree centrality. As far as authorities are concerned, eigencentrality stresses the indirect links with important actors. This is the case of Frank, who is at the core of the company.

The union network From the union of the networks related to the first four questions, that is the binarized sum of matrices $Q_{i}^{b}(i=1, \ldots, 4)$, all direct connections among actors can be detected. The level of communication, or relational density, is high: $48 \%$, i.e. the number of direct ties (115) out of 240 possible connections. In other words, each node is on average directly connected to eight other nodes for one reason or another (see Figs. 4, 5 for in- and out-degree rankings for the networks associated to $Q_{i}(i=1, \ldots, 4)$ and their union.

From Fig. 4, Charly and Frank are good leaders within the organization. Chin is good for first hand help (question $Q_{1}$ ) and for creation of value (question $Q_{4}$ ). Taggy and Coch are peripheral, even though Taggy may be of help in some situations. A closer check at the univariate networks shows that Frank has good scores for the first three univariates, $Q_{1}, Q_{2}$ and $Q_{3}$, but not for $Q_{4}$. So he probably does not help in saving time and neither does Ted.

An analysis of the rankings of out-degrees (Fig. 5) shows interesting insights. Ted asks a lot. Also Jac is an asker being a newcomer to the organization.

Summing up the results in multiple networks, Charly is concluded to be the driver: he gives advice at all levels, from simple information to substantial help. Also Frank is a good leader, but has less charisma than Charly. Alex should enhance her level of communication. Joan should be helped in improving her self image. 


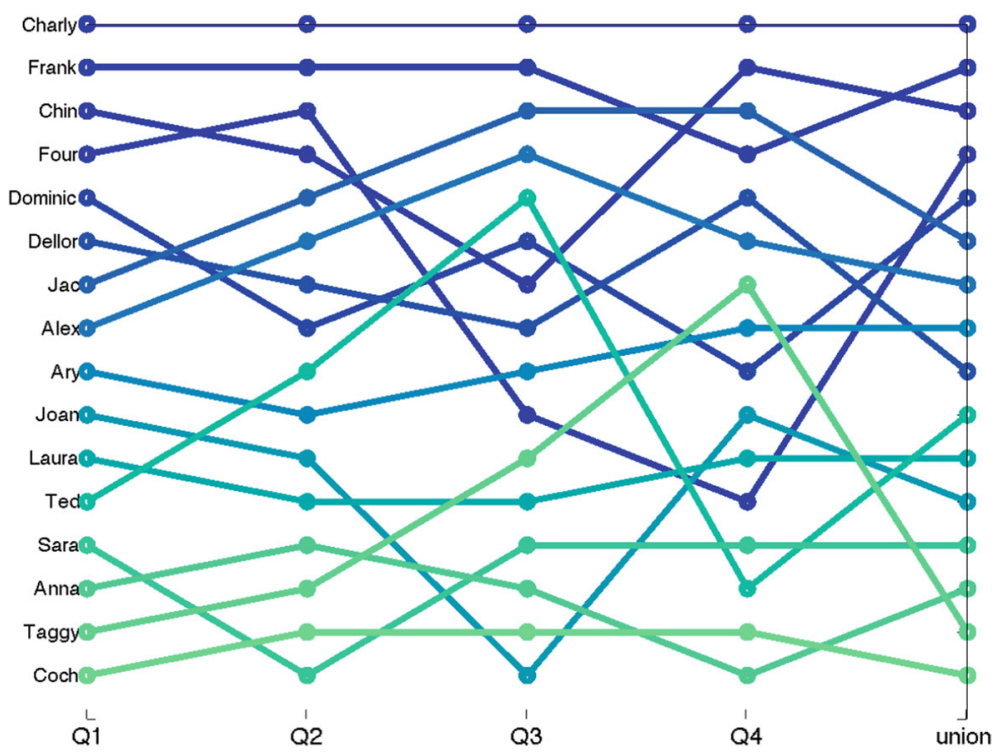

Fig. 4 Union: in-degree rankings

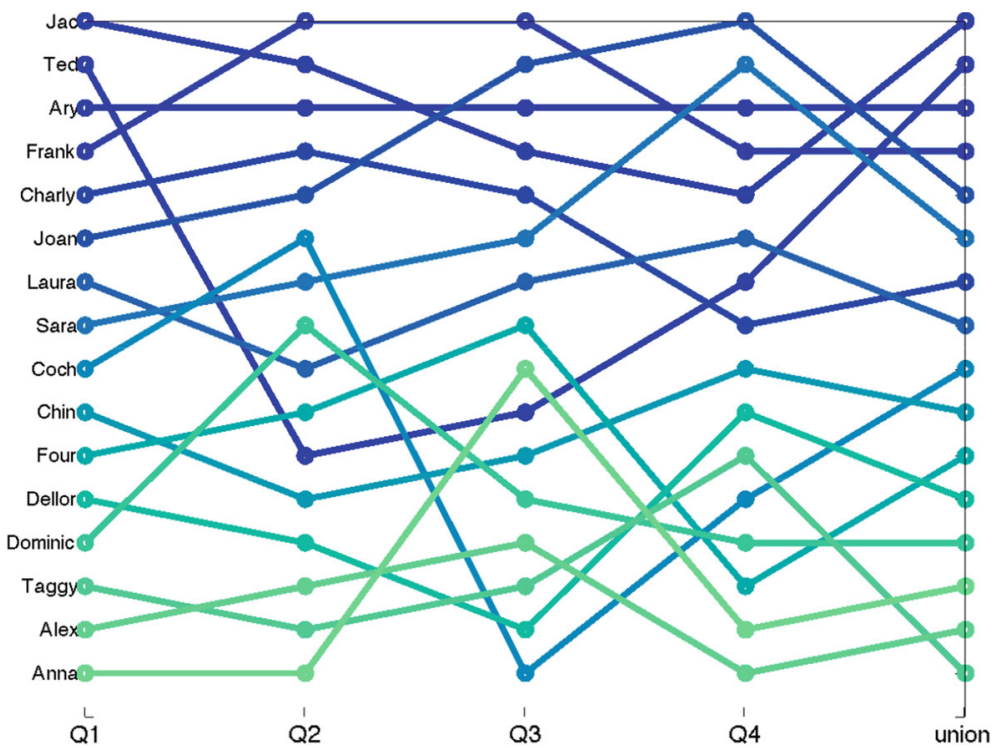

Fig. 5 Union: out-degree rankings

Mutual awareness To check for mutual awareness, for each $Q_{i}(i=1, \ldots, 4), Q_{i+5}$ the related binary adjacency matrices are considered. The comparison of the two matrices allow to reveal levels of poor communication between individuals. In fact, both questions $Q_{i}$ and $Q_{i+5}$ correspond to the same type of relation from the perspective of who contacts and who is contacted. Clearly, individuals with a high hubness score in $Q_{i}$ are expected to have an equally high authority score in $Q_{i+5}$ and vice versa, otherwise their role is not properly 


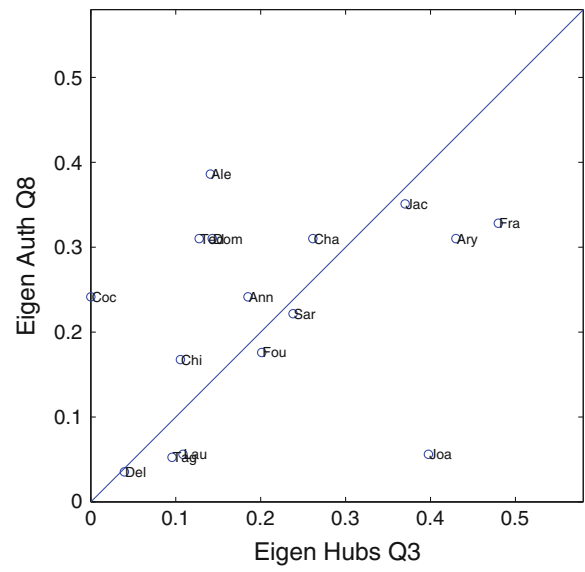

(a)

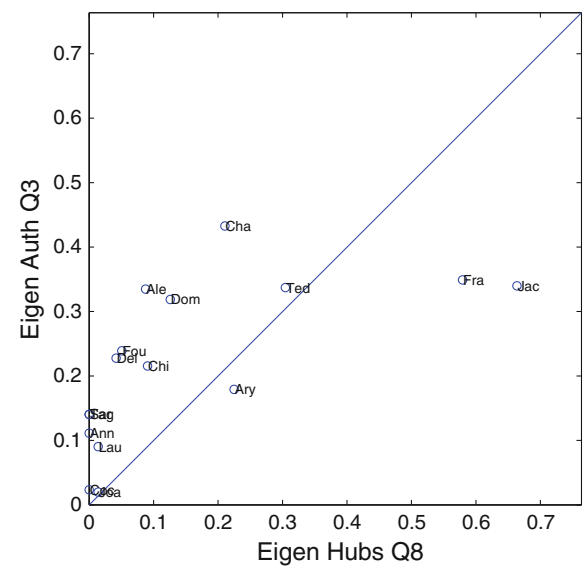

(b)

Fig. 6 Bisecting lines for $Q_{3}$ and $Q_{8}$

perceived by the others. Hubs and authority centralities were computed and plotted on an $x-y$ plane. The results related to the networks associated to questions $Q_{3}$ and $Q_{8}$ are hereby discussed.

In Fig. 6a, equilibrium is found for individuals on the bisecting line (Jac and Charly in particular). Individuals positioned below the bisecting line ask colleagues for help in making decisions, but colleagues do not perceive it. Those individuals show personal communication problems such as shyness. This is the case of Ary and Joan. Ary, a peripheral specialist, asks for information and help, but the others do not perceive it properly. Ted and even more so Alex, with a hub score half of the authority score, claim to ask rarely, but others perceive it differently. They do not admit their need for information.

In Fig. 6b, individuals positioned below the bisecting line claim to help colleagues by making decisions more so than their colleagues perceive it. Those individuals overvalue their roles in the organization and there is obviously a communication problem. This is the case of Jac, Frank and Ary. Charly and Frank are in an almost specular position: Frank claims he gives help in making decisions while others do not perceive it. On the other hand, Charly gives more advice than he claims to do. Ted is in equilibrium and gives sound advice.

Global mutual awareness In order to have a quantitative assessment of the mutual awareness at a global level, i.e. referring to the organization as a whole, a procedure known as QAP (see Krackhardt 1987, 1988, 1992b; Hanneman and Riddle 2005; Chen et al. 2007 for an application), designed to compare statistical arrays of data, is performed. The pairs $\left\{Q_{i}^{w},\left(Q_{i+5}^{w}\right)^{T}\right\}$, with $i=1,2,3,4$, are considered and the correlation coefficient $r_{i, i+5}$ between the elements $(k, l)$ of $Q_{i}^{w}$ and the elements $(l, k)$ of $Q_{i+5}^{w}$, i.e. the correlation coefficient between two networks with the same actors is computed. In order to assess the degree of correlation between the two sets of data, the observed values were compared with the null distribution of the correlation coefficients. The null distribution is obtained first by scrambling the entries of $Q_{i+5}^{w}$, applying the same permutation to both rows and columns. In such a way, the structure of the network remains unaltered, since the resulting graphs are isomorphic. By performing such permutations a large number of times (10 6 in our case) and computing for each permutation the correlation coefficient, a proxy of the null distribution of the correlation coefficient was obtained. The proportion of correlation coefficients computed 


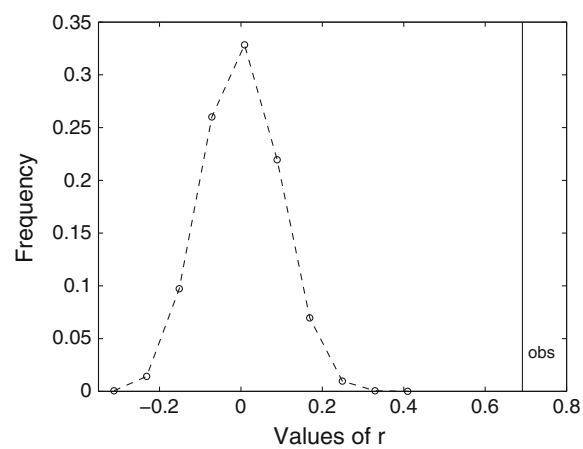

(a) $Q_{1}$ vs $Q_{6}^{T}$

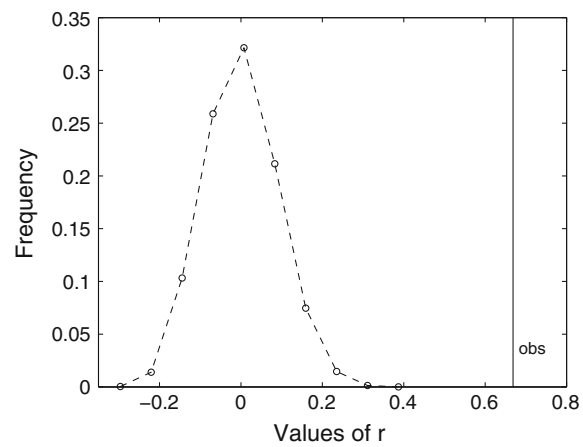

(c) $Q_{3}$ vs $Q_{8}^{T}$

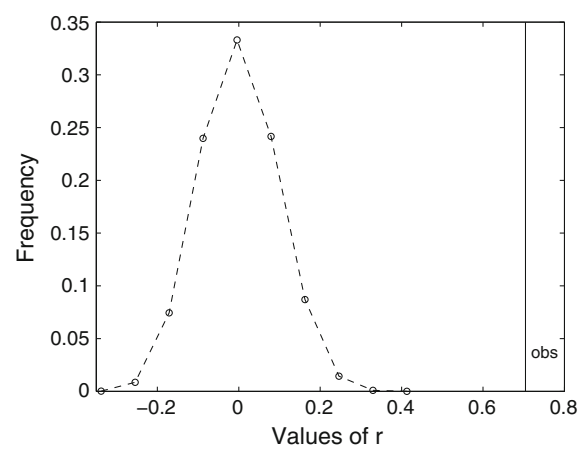

(b) $Q_{2}$ vs $Q_{7}^{T}$

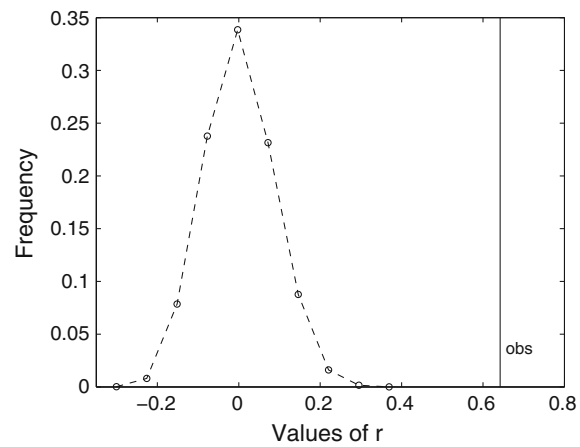

(d) $Q_{4}$ vs $Q_{9}^{T}$

Fig. 7 Correlation coefficient sampling QAP distribution (dotted line) versus the observed values (continuous vertical line)

Table 1 Observed versus QAP correlation coefficients (statistics)

\begin{tabular}{llclc}
\hline & Observed $r$ & Mean $r(\mathrm{QAP})$ & StDev of $r(\mathrm{QAP})$ & $P$ \\
\hline$Q_{1}^{w}$ vs $\left(Q_{6}^{w}\right)^{T}$ & 0.6915 & -0.000067 & 0.0906 & 1 \\
$Q_{2}^{w}$ vs $\left(Q_{7}^{w}\right)^{T}$ & 0.7044 & 0.000046 & 0.0938 & 1 \\
$Q_{3}^{w}$ vs $\left(Q_{8}^{w}\right)^{T}$ & 0.6686 & -0.000073 & 0.0889 & 1 \\
$Q_{4}^{w}$ vs $\left(Q_{9}^{w}\right)^{T}$ & 0.6420 & -0.000103 & 0.0847 & 1 \\
\hline
\end{tabular}

from the permuted data that are at least as large as the observed statistics, represents the $p$ value of the test.

Figure 7 reports the sampling QAP distribution of the correlation coefficient (dotted line) and the empirical value (continuous vertical line). The proportion of correlation coefficients that are at least as large as the observed values is one (Table 1), allowing the acceptance of the hypothesis that the pairs of networks with weighted adjacency matrix $Q_{i}^{w}$ and $\left(Q_{i+5}^{w}\right)^{T}$ are correlated, suggesting a high degree of global mutual awareness. In the second column of Table 1, the observed values of the correlation coefficient are reported for each couple of matrices. The mean and the standard deviation of the correlation coefficients obtained with QAP are in the third and fourth column. The last column $(P)$ reports the proportion of QAP trials that produce a correlation coefficient as large as the observed correlation coefficient. 
In conclusion, a high degree of global mutual awareness has been found, suggesting that the organization as a whole is efficient and the level of communication is generally good.

\section{Conclusions}

The analysis conducted on the case study has shown the following:

- A high degree of communication is detected within the organization, flowing at all levels, from simple information requests to help in making decisions.

- A high level of mutual awareness suggests good relationships among people, which greatly contributes to the efficiency of the organization.

- At the individual level, Charly and Frank are recognized as good information brokers. This is particularly important since they occupy managing positions. Charly is recognized as the driver: he gives help and advices even more than he claims to do. Frank is also recognized as a driver, but he should try to be more open towards people and accept sharing responsibilities with others. Alex should try to involve others in her decision making, in order to enhance her role. Ted takes an aside position, even though he is still seen as the original leader.

- Some work has to be done on an individual level for Joan, one of the secretaries. Ary is a peripheral specialist and its expertise should be enhanced and valorized. He should be more involved at all levels and helped in sharing knowledge and acquaintance.

The results obtained analyzing the informal networks were subsequently compared with the formal structure. In general, the role of the formal leaders in the organization is recognized also at an informal level and the flow of communication is good. Charly in particular can be the leader after transition.

In this paper, some interesting results have been obtained. As far as the case study is concerned, the informal leaders (for information, help and advice) were discovered and critical positions were discussed. This approach can be successfully extended to other practical cases.

Our research contributes to SNA by introducing centrality for hubs and authorities applied to multiple networks and the concept of mutual awareness.

Furthermore, our results can provide support for improving the efficiency of the organization through a better level of communication and mutual understanding of each other's needs.

Acknowledgements We thank the anonymous referees for their valuable suggestions that improved our paper substantially.

\section{References}

Anklam, P.: Knowledge management: the collaboration thread. Bull. Am. Soc. Inf. Sci. Technol. 28(6), 8-11 (2002)

Anklam, P.: KM and the social network. Knowl. Manag. Mag., 24-28 (2003)

Bonacich, P.: Power and centrality: a family of measures. Am. J. Sociol. 92, 1170-1182 (1987)

Bonacich, P.: Some unique properties of eigenvector centrality. Soc. Netw. 29, 375-395 (2007)

Brass, D.J.: Being in the right place: a structural analysis of individual influence in an organization. Adm. Sci. Q. 29, 518-539 (1984)

Chen, M., Su, K., Tsai, W.: Competitive tension: the awareness-motivation-capability perspective. Acad. Manag. J. 50, 101-118 (2007) 
Cross, R.: The Hidden Power of Social Networks: Understanding How Work Gets Really Done in an Organization. Harvard Business School Press, Boston (2004)

Cross, R., Cummings, J.N.: Tie and network correlates of individual performance in knowledge intensive work. Acad. Manag. J. 47, 928-937 (2004)

Cross, R., Prusak, L.: The people that make organizations go - or stop. Harv. Bus. Rev. 80(6), 104-112 (2002)

Cross, R., Sproull, L.: More than an answer: informative relationship for actionable knowledge. Organ. Sci. 15, 446-462 (2004)

Cross, R., Thomas, R.J.: How top talent uses networks and where rising stars get trapped. Organ. Dyn. 37(2), 165-180 (2008)

Cross, R., Borgatti, S.P., Parker, A.: Beyond answers: dimensions of the advice network. Soc. Netw. 23, 215-235 (2001)

Cross, R., Borgatti, S.P., Parker, A.: Making invisible work visible: using social network analysis to support strategic collaboration. Calif. Manag. Rev. 44, 25-46 (2002)

De Toni, A.F., Nonino, F.: The key roles in the informal organization: a network perspective analysis. Learn. Organ. 10(17), 86-103 (2010)

Farahat, A., Lofaro, T., Miller, J.C., Rae, G., Ward, L.A.: Authority rankings from HITS, PageRank and SALSA: existence, uniqueness and effect of initialization. SIAM J. Sci. Comput. 27(4), 1181-1201 (2006)

Freeman, L.C.: A set of measures of centrality based on betweenness. Sociometry 40, 35-41 (1977)

Gibbons, D.E.: Friendship and advice networks in the context of changing professional values. Adm. Sci. Q. 49, 238-262 (2004)

Grassi, R., Stefani, S., Torriero, A.: Some new results on the eigenvector centrality. J. Math. Sociol. 31, 237-248 (2007)

Grassi, R., Scapellato, R., Stefani, S., Torriero, A.: Betweenness centrality: extremal values and structural properties. In: Naimzada, A.K., Stefani, S., Torriero, A. (eds.) Networks, Topology and Dynamics-Theory and Applications to Economics and Social Systems, pp. 161-176. Lecture Notes in Economics and Mathematical Systems, vol. 613. Springer, Heidelberg. ISBN: 978-3-540-68407-7 (2009)

Grassi, R., Stefani, S., Torriero, A.: Centrality in organizational networks. Int. J. Intell. Syst. 3, 253-265 (2010a)

Grassi, R., Stefani, S., Torriero, A.: Extremal properties of graphs and eigencentrality in trees with a given degree sequence. J. Math. Sociol. 34(2), 115-135 (2010b)

Hanneman R.A., Riddle M.: Introduction to social network methods. Published in digital form at http://faculty. ucr.edu/ hanneman/ (2005). Accessed 10 Sept 2011

Harary, F.: Graph Theory. Addison-Wesley, Reading (1969)

Kleinberg, J.M.: Authoritative sources in a hyperlinked environment. J. ACM 46(5), 604-632 (1999)

Krackhardt, D.: QAP partialling as a test of spuriousness. Soc. Netw. 9, 171-186 (1987)

Krackhardt, D.: Predicting with networks: non parametric multiple regression analysis of dyadic data. Soc. Netw. 10, 359-381 (1988)

Krackhardt, D.: The strength of strong ties: the importance of Philos in organizations. In: Nohria, N., Eccles, R.G. (eds.) Networks and Organizations: Structure, Form and Action, pp. 216-239. Harvard University Press, Boston (1992a)

Krackhardt, D.: A caveat on the use of the Quadratic Assignment Procedure. J. Quant. Anthropol. 3, 279-296 (1992b)

Krackhardt, D., Hanson, J.R.: Informal networks: the company behind the chart. Harv. Bus. Rev., July-August, 104-111 (1993)

Krackhardt, D., Stern, R.: Informal networks and organizational crises: an experimental simulation. Soc. Psychol. Q. 51, 123-140 (1988)

Naddaf, Y., Mutyala, S.: Social network analysis and community mining in organizations based on email records, http://www.asymmetricventures.com/files/2010/08/asymmetric_ventures_sna_white_ paper.pdf (2010). Accessed 1 March 2012

Naimzada, A.K., Stefani, S., Torriero, A. (eds.): Networks, Topology, and Dynamics-Theory and Applications to Economics and Social Systems. Lecture Notes in Economics and Mathematical Systems, vol. 613. Springer, Heidelberg (2009). ISBN: 978-3-540-68407-7

Newman, M.E.J.: Networks-An Introduction. Oxford University Press, Oxford (2010)

Okada, A., Imaizumi, T.: A generalization of two-mode three-way asymmetric multidimensional scaling. In: Gaul, W., et al. (eds.) Classification, Automation and New Media, pp. 115-122. Springer, Berlin (2002)

Okada, A., Imaizumi, T., Inoue, H.: Asymmetric multidimensional scaling of relationships among managers of a firm. In: Baier, D., Decker, R., Schmidt-Thieme, L. (eds.) Data Analysis and Decision Support, Studies in Classification, Data Analysis, and, Knowledge Organization, pp. 100-107. Springer, Berlin (2005) 
Okada, A., et al.: Two dimensional centrality of asymmetric social networks. In: Palumbo, F. (ed.) Data Analysis and Classification-Studies in Classification, Data Analysis, and Knowledge Organization, pp. 93-100. Springer, Berlin (2010)

Reagans, R., Zuckerman, E., McEvily, B.: How to make the team: social networks vs. demography as criteria for designing effective teams. Adm. Sci. Q. 49, 101-133 (2004)

Robins, G., Pattison, P.: Multiple networks in organizations. http://www.sna.unimelb.edu.au/publications/ multiple_networks.pdf (2006). Accessed 1 Oct 2012

Stefani, S., Torriero, A.: Formal and informal networks in organizations. Stud. Comput. Intell. 448, 61-67 (2013)

Wassermann, S., Faust, K.: Social Network Analysis: Methods and Applications. Cambridge University Press, New York (1994) 